Review

\title{
Computer vision and artificial intelligence in precision agriculture for grain crops: A systematic review
}

\author{
Diego Inácio Patrício ${ }^{\mathrm{a}, *}$, Rafael Rieder ${ }^{\mathrm{b}}$ \\ a Brazilian Agricultural Research Corporation, Embrapa, Passo Fundo, Rio Grande do Sul, Brazil \\ ${ }^{\mathrm{b}}$ Graduate Program in Applied Computing (PPGCA), Institute of Exact Sciences and Geosciences (ICEG), University of Passo Fundo (UPF), Passo Fundo, Rio Grande do \\ Sul, Brazil
}

\section{A R T I C L E I N F O}

\section{Keywords:}

Computer vision

Artificial intelligence

Precision agriculture

Systematic review

2018 MSC:

00-01

99-00

\begin{abstract}
A B S T R A C T
Grain production plays an important role in the global economy. In this sense, the demand for efficient and safe methods of food production is increasing. Information Technology is one of the tools to that end. Among the available tools, we highlight computer vision solutions combined with artificial intelligence algorithms that achieved important results in the detection of patterns in images. In this context, this work presents a systematic review that aims to identify the applicability of computer vision in precision agriculture for the production of the five most produced grains in the world: maize, rice, wheat, soybean, and barley. In this sense, we present 25 papers selected in the last five years with different approaches to treat aspects related to disease detection, grain quality, and phenotyping. From the results of the systematic review, it is possible to identify great opportunities, such as the exploitation of GPU (Graphics Processing Unit) and advanced artificial intelligence techniques, such as DBN (Deep Belief Networks) in the construction of robust methods of computer vision applied to precision agriculture.
\end{abstract}

\section{Introduction}

Grain consumption has grown in recent years as well as growing interest in efficient and sustainable agricultural processes in order to fit consumer demand. According to data from the Food and Agriculture Organization of the United Nations (FAO) (Food, 2012), only in 2014 were harvested 2.9 billion tonnes of the five main grains grown in the world in order of productivity: corn, rice, wheat, soy, and barley.

In agriculture is noteworthy that image processing and computer vision applications have grown due to reduced equipment costs, increased computational power, and increasing interest in non-destructive food assessment methods (Mahajan et al., 2015). The use of these techniques presents advantages when compared with traditional methods based on manual work, however, there are still a number of challenges to be overcome (Barbedo, 2016).

The machine vision usage has grown in recent years to meet the growing demand for fast and accurate methods in monitoring grain production. An example, according to Zareiforoush et al. (2015) was the use of such methods in rice production to raise the quality of the final product and to fit food safety criteria in an automated, economically efficient, and non-destructive way.
Manual methods for grain assessment are challenging even for people who are trained to perform these tasks. Although, one of the main difficulties is the training of these evaluators. There are scarce places prepared to train people with the necessary quality. Another difficulty is the time required to carry out such evaluations, which prevents quickly decision making and large-scale evaluation.

The use of computer vision, near-infrared spectroscopy, magnetic resonance spectroscopy, electronic nose, spectroscopy using the Fourier transform in infrared light, X-ray and hyperspectral images are some of the techniques that can be used to overcome these limitations (Vithu and Moses, 2016). Such techniques, combined with pattern recognition algorithms and automatic classification tools, have been used to deal with the challenge of monitor cultures and analyze food quality.

Machine Learning Algorithms enable you to analyze massive volumes of data, regardless of complexity, quickly and accurately. Its use is already common in many areas such as fraud detection, credit analysis, fault prediction models, image recognition patterns, intelligent spam filters and product quality analysis. However, considering the variety of alternatives, it is essential to know the individual characteristics of each method and the best scenario for its use.

One of the main factors in popularizing the machine learning usage

\footnotetext{
* Corresponding author.

E-mail addresses: diego.patricio@embrapa.br (D.I. Patrício), rieder@upf.br (R. Rieder).

URLS: http://www.embrapa.br/trigo (D.I. Patrício), http://www.upf.br (R. Rieder).
} 


\begin{tabular}{ll} 
Nomenclature \\
ACM & Association for Computing Machinery \\
ANN & Artificial Neural Network \\
BIC & Border/Interior Classification \\
BOV & Bag of Visual words \\
BPNN & Back-Propagation Neural Network \\
CCV & Color Coherence Vector \\
CDH & Color Difference Histogram \\
CNN & Convolution Neural Network \\
CPU & Central Process Unit \\
DBN & Deep Belief Network \\
DBN & Deep Belief Networks \\
DOM & Degree of Milling \\
DS & Decorrelation Stretching \\
DSIFT & Dense Scale-invariant Feature Transform \\
ExGExR & Excess Green - Excess Red \\
FAO & Food And Agriculture Organization of the United Nations \\
FV & Fisher Vector \\
GLCM & Gray Level Co-occurrence Matrix \\
GPU & Graphic Processing Unit \\
GW & Gabor Wavelet \\
HOG & Histogram of Gradients \\
HSI & Hue, Saturation and Intensity \\
HSI & Hyperspectral Imaging \\
ICA & Imperialist Competitive Algorithm \\
IEEE & Institute of Electrical and Electronic Engineers \\
PLS-DA & Partial Least Squares Discriminant Analysis \\
KNN & k-Nearest Neural Network \\
LAP & Localized Angular phase \\
\hline
\end{tabular}

was the large-scale use of GPUs (Graphic Processing Units). This type of specialized hardware initially developed to meet the rendering needs of digital games, has evolved to expand its use in a generic way in diverse areas such as industry, health, climate, computational modeling. They have high parallel processing capacity due to the presence of high amount of processors cores. Thus, they can offer far superior performance when compared to CPUs (Central Process Unit) depending on the type of process. In addition, the advent of the CUDA programming language, and the inclusion of dedicated hardware to facilitate the parallel programming of these processors help developers in building new applications. In this way, it is possible through the use of GPUs to obtain better solutions to a given problem in a shorter period of time (Kirk and Hwu, 2016).

The union of these three components: computer vision, machine learning, and high-performance computing have been shown to be promising in solving different problems in agriculture. The predictive potential made possible by deep learning, a technique that has gained prominence among the others used in machine learning, will cause a disruptive effect in different segments of the traditional industry as well as agriculture.

Due to the relevance of the subject, several revisions have been produced over the last years with the objective of analyzing the published works available in the literature. Barbedo (2013) presented a review of digital image processing techniques to detect, quantify and classify plant diseases from digital images. Those methods include detection, classification, and quantification. Techniques like support vector machines, fuzzy logic, and neural networks are also evaluated. Zareiforoush et al. (2015) published a systematic review of the opportunities to use computer vision in rice quality inspection. The work focused on the practical aspects of rice processing like head rice yield measurement, the degree of milling, fissure identification, shape and size analysis, color analysis, variety classification, chalkiness and internal damage assessment, root estimation, and spikelet analysis. Vithu

$\begin{array}{ll}\text { LBP } & \text { Local Binary Pattern } \\ \text { LED } & \text { Light Emit Diode } \\ \text { MCW } & \text { Marker Controlled Watershed } \\ \text { METS } & \text { Minimum Error Threshold Selecting } \\ \text { MLP } & \text { Multi Layer Perceptron } \\ \text { MSER } & \text { Maximally Stable Extremal Regions } \\ \text { NNI } & \text { Neural Network Intensity } \\ \text { PBK } & \text { Percentage of Broken Kernels } \\ \text { PCA } & \text { Principal Component Analysis } \\ \text { PHOW } & \text { Pyramid histograms of visual words } \\ \text { iPLS-DA } & \text { Interval Partial Least Squares Discriminant Analysis } \\ \text { PNN } & \text { Probabilistic Neural Network } \\ \text { PSR } & \text { Pitch Segmentation Recognition } \\ \text { RGB } & \text { Red, Green and Blue } \\ \text { RMSECV } & \text { Root Mean Square Errors of Cross Validation } \\ \text { SEH } & \text { Strutucture Element Histogram } \\ \text { SIFT } & \text { Scale-invariant Feature Transform } \\ \text { SLC } & \text { Saliency Color Space } \\ \text { SLIC } & \text { Simple Linear Iterative Clustering } \\ \text { SMH } & \text { Support Vector Machine, Maximally Stable Extremely } \\ & \text { Regions and Histograms of Oriented Gradient Method } \\ \text { SPA } & \text { Successive Projection Algorithm } \\ \text { SSLBP } & \text { Square Symmetric Local Binary Pattern } \\ \text { SURF } & \text { Speeded-Up Robust Features } \\ \text { SVM } & \text { Support Vector Machine } \\ \text { VFD } & \text { Volume Fractal Dimension } \\ \text { VFDCA } & \text { Volume Fractal Dimension with Canonical Analysis } \\ \text { WDH } & \text { Wavelet Decomposed Color Histogram } \\ \text { YCbCr } & \text { Luma, Blue-difference and Red-difference Chroma com- } \\ & \text { ponents } \\ & \end{array}$

and Moses (2016) analyzed published works related to computer vision systems applied in food grain quality evaluation. Among the applications analyzed, this review analyses aspects like identification of foreign matter, insect infestation, microbial infection, and discolored grains. Shah et al. (2016) presents a survey of different image processing and machine-learning techniques used in the identification of rice plant diseases based on images of infected rice plants. The survey is organized in two main parts. The first one deals with image processing tasks like acquisition, preprocessing, segmentation, and feature extraction whereas the second part deals with machine learning tasks. Barbedo (2016) addressed the main challenges in automatic disease identification. Among the topics discussed are the intrinsic and extrinsic factors of the image that could affect the performance of the automatic identification techniques. Kamilaris and Prenafeta-Boldú (2018) review the efforts to employ deep learning techniques in agricultural and food production challenges pointing out that those methods provide high accuracy and outperforms commonly used image processing techniques.

In contrast of the reviews presented earlier, this survey aimed to study how machine vision can be applied to the main cultures of grains in a broad aspect and which techniques can operate in combination with artificial intelligence. The idea is to evaluate, through a systematic review, challenges and techniques of computer vision and artificial intelligence applied in diseases identification and pests infestations detection, grain quality, and phenotyping and phenology. In addition, this review intends to identify gaps and opportunities in order to be an updated reference for future works.

\section{Methodology}

The present systematic review aims to identify in the literature works related to the use of computer vision and artificial intelligence techniques for the five largest grain crops, considering the total tonnes 
harvested for these crops in 2014 (Food, 2012). In this sense, a protocol was previously established to identify, evaluate and interpret results relevant to these research scope using an explicit and systematic method of literature analysis (Kitchenham and Charters, 2007).

Five databases were used for the analysis of the literature: ScienceDirect (ScienceDirect, 2018), Scopus (Scopus, 2018), Springer (Springer Link, 2018), Web of Science (Web of Science, 2018), ACM (Association for Computing Machinery) (ACM Digital Library, 2018) and IEEE (Institute of Electrical and Electronic Engineers) (IEEE Xplore Digital Library, 2018). For the survey of papers, the following search expression was defined: ("computer vision" OR "machine vision" OR "artificial intelligence" OR "machine learning" OR "deep learning" OR "GPU" OR "Graphics Processing Unit") AND (maize OR rice OR wheat OR soybean OR barley). The time interval used in the research was defined from 2013 to 2017, that is, last 5 years. The studies considered in this review were published in English in periodicals, disregarding chapters of books, proceedings, and summaries of events and seminars.

The evaluation of the work was carried out in three stages: identification, screening, and eligibility. In the identification stage, the search expressions are used to obtain the works of interest. The results of the different databases were consolidated for the removal of duplicate items. In the triage stage, the title and the abstract of the work were verified if it meets the area of interest of the present review. At the eligibility stage, it was assessed whether the paper met the eligibility criteria and whether the results and the conclusion were satisfactory. The quality of the description of the technique under review and the comparison with other similar methods were also considered. For each eligible study, the following information was obtained: authors, year of publication, general objectives, description of the method used for image processing, description of the method used for image classification using artificial intelligence, and if GPU was used.

In the "Identification" stage 1578 non-duplicate items were obtained using metadata filtering when this feature was available. In the next stage, "Screening", the titles and abstracts of the papers were analyzed and 183 items were selected for the integral reading and verification of results. In the final stage of the systematic review, the eligibility criteria for the work were verified. Of the 183 papers obtained in the previous stage, 25 were selected to be included in this systematic review.

\section{A gentle introduction to computer vision and machine learning}

\subsection{Computer vision}

Computer Vision comprehends methods and techniques through which artificial vision systems can be constructed and employed in a reasonable way in practical applications. This area of computer science includes the software, hardware, and imaging techniques necessary those methods (Davies, 2005).

Basically, a computer vision system is composed of the following stages: image acquisition and image processing.

- Image Acquisition: Starts by the transferring of electronic signals from a sensor to a numerical representation by a device like a camera (Zareiforoush et al., 2015). Mainly, two types of cameras distinguished by the form of scanning can be used: area or line. Conventional or area-scanning cameras generates an image in each exposure cycle. In contrast, line scan cameras capture only one line of pixels at a time. For the acquisition of two-dimensional images, it is necessary to move the object to be captured using a conveyor or move the camera along a stationary object. The quality of the image acquired by a computer vision system is directly affected by the illumination used during the acquisition phase. In this way, all the effort invested in the use of adequate lighting will increase the performance and reliability of the system, reducing the complexity of the software used in the processing stage (Hornberg, 2017).
- Image Processing: Involves tasks performed to manipulate digital images for the purpose of, for example, improving their quality, reducing noise or correcting lighting problems. In addition, image analysis refers to the process to differentiate regions of interest from the other regions to extract information. Can be divided in low-level, intermediate-level and high-level processing. Low-level processing, or preprocessing, includes operations to grayscale adjustment, focus correction, contrast or sharpness enhancement and noise reduction. Those operations produce a new image and are employed to improve the image quality or to modify the position of the object of interest by geometric transformations (Hornberg, 2017). Meanwhile, middle-level processes involve segmentation operations (partitioning of images in regions), description and classification of objects present in the image. Image segmentation results in a set of contours or regions. The extraction of attributes that characterize these regions is necessary to evaluate a series of characteristics of the region of interest. For example, the ellipse parameters allow you to determine the orientation and size of the region. The bounding box allows the calculation of the height and width of the region of interest (Schaeffel, 2017). Filters such as Sobel, Laplacian, and Laplacian of Gaussian can be used to determine regions where abrupt changes in intensity occur in the image. The Gabor filter is selective for elements in the image within a certain range of orientation and frequency. Haar type filters consist of adjacent rectangular regions that are balanced where the mean value of the filter is zero and invariant to illumination changes. The purpose of edge detection is to produce a binary image where nonzero values denote the presence of an edge in the image. Detectors may optionally also return other information such as scale and orientation relative to an edge. Some examples of edge detectors are Canny, Harris, and SIFT. The SIFT (Scale Invariant Feature Transform) detector is a method for identifying points of interest. It associates scale and orientation information for each point resulting from the detection process (Prince, 2012). High-level processes, or image classification, involve the recognition and classification of regions of interest that are usually performed by statistical classifiers or neural networks.

\subsection{Machine learning}

Machine learning deals with methods and techniques for computational applications capable of modifying or adapting their actions, in order to make them more precise. It is possible to classify learning algorithms in the following categories: supervised learning, unsupervised learning, reinforcement learning, and evolutionary learning.

Supervised learning algorithms are those that start from a set of correct answers (training set) to generalize and respond correctly to all possible inputs. In contrast, unsupervised learning algorithms compare inputs to each other to identify similarities in order to classify them into categories. Reinforcement learning algorithms can be classified as an intermediary stage between supervised and unsupervised. The algorithm is informed when it responds incorrectly to a question, but this does not happen when it responds correctly. You have to explore the different answers until the correct answers are found. Finally, evolutionary learning models the learning process of microorganisms, which consists of adapting to increase the chances of surviving and reproducing in the environment in which they live. Both regression and classification problems can be treated with machine learning (Marsland, 2014).

The machine learning process consists of the following steps (Marsland, 2014):

- Data Preparation and Acquisition: Initial step that consists in obtaining the appropriate data that contains characteristics to be considered in learning. In general, learning algorithms require a large amount of data. It is also important to note that the amount of data is a factor to be considered. Balancing the demand for large 
amounts of data, preferably without much noise, with the computational cost that increases proportionally with the amount of data is a relevant point to consider.

- Selecting Characteristics of Interest: is to identify the most significant characteristics for the problem that will be addressed.

- Algorithm Selection: Consist into select the most appropriate algorithm to treat the problem being studied.

- Parameters Selection: Some algorithms need to be tuned by parameters that require experimentation to be defined.

- Training: Given a set of inputs, the algorithm, and the parameters, the training consists of the construction of the computational model that will be used in the prediction of the answers to new data.

- Rating: The system needs to be evaluated for its accuracy for the data it has been trained.

\subsubsection{Support Vector Machines}

SVM (Support Vector Machines) is a non-probabilistic linear classifier that constructs a decision threshold with the greatest possible distance between the example points. A classic SVM classifier is able to classify the points of the input set into two possible classes. Separation thresholds, although linear, have the capacity to incorporate data into a higher space. In this way, it is possible to create linear separators even if in the original space of the inputs it is impossible to define it linearly. Finally, SVM is a non-parametric method. Therefore, it has the flexibility to represent complex functions while at the same time being resilient to overfitting (Russell and Norvig, 2009).

\subsubsection{Neural networks}

A neural network is composed of connected nodes by directed links with an associated weight that determines the strength and the signal of the connection. Weights are the primary form of long-term storage in neural networks and learning algorithms can usually update these weights. Input and output nodes are those with connections to the external world. Each node is independent of the others and, thus, can perform its calculations from only its input values and respective weights. Each unit receives the values from the incoming links, calculates the activation level and, when activated, sends the signal to all its outgoing links (Russell and Norvig, 2009).

A feedforward Neural Network has connections in only one direction and can be represented as a directed acyclic graph. Each node receives inputs from the upstream nodes and delivers the output to the downstream nodes without cycles. This kind of network represents a function and has no internal state except the link weights. In contrast, a recurring neural network feeds the input nodes with their outputs. In this way, the network forms a dynamic system that may or may not reach a steady state (Russell and Norvig, 2009).

Neural networks are organized in layers where each unit receives input from only the nodes of the next higher layer. In networks that have one or more layers, there are called hidden units for those that are not directly connected to the network output. With only a sufficiently large hidden layer it is possible to represent any continuous function with arbitrary accuracy. With two layers, even non-continuous functions can be represented. For the learning algorithm, a set of examples and a neural network are necessary. The final will result in a trained network with adjusted weights capable of processing an input and producing an output with the computed data class (Russell and Norvig, 2009).

\subsubsection{Deep learning}

Deep Learning Networks differ from conventional Neural Networks in the sense that they have more nodes, more complex means of layer interconnection, they require strong computational power for their training and they have automatic extraction of the parameters.

The four main architectures used are: Unsupervisioned Pretrained Networks, Convolutional Neural Networks, Recurrent Neural Network, Recursive Neural Network. These types of networks are already used in the solution of various problems such as text-to-speech conversion, cite fan2014synthesis, language identification (Sak et al., 2014), translation (Sutskever et al., 2014), signal processing (Marchi et al., 2014), automatic recognition of characters (Breuel et al., 2013), automatic generation of subtitles (Zaremba et al., 201), among others. Mainly, in image classification, convolutional neural networks are the most used because of your nature. The primary purpose of a Convolutional Neural Network or Convet is to learn data characteristics from convolution operations. Therefore, this type of network is better suited for pattern recognition in images.

LeNet-5 was one of the first proposed architectures for a ConvNet (Lecun et al., 1998). This network architecture consists of three types of

Table 1

Resume of the eligible papers into systematic review

\begin{tabular}{|c|c|c|c|c|c|c|c|}
\hline$\#$ & References & Year & Group & Crop & Device & Target & Classifier \\
\hline 1 & Chung et al. (2016) & 2016 & Diseases & Rice & Scanner & Seedlings & SVM \\
\hline 2 & Shrivastava et al. (2016) & 2016 & Diseases & Soybean & Camera & Leaves & SVM \\
\hline 3 & Liu et al. (2016) & 2016 & Diseases & Wheat & Camera & Field & SVM \\
\hline 4 & Pires et al. (2016) & 2016 & Diseases & Soybean & Scanner & Leaves & SVM \\
\hline 5 & Han et al. (2015) & 2015 & Diseases & Wheat & Camera & Field & SVM \\
\hline 6 & Boniecki et al. (2014) & 2014 & Diseases & Wheat & Scanner & X-ray & MLP \\
\hline 7 & Sabanci et al. (2017) & 2017 & Quality & Wheat & Camera & Kernels & ANN \\
\hline 8 & Sun et al. (2016) & 2016 & Quality & Rice & Camera & Kernels & DBN \\
\hline 9 & Liu et al. (2016) & 2016 & Quality & Rice & Camera & Kernels & SVM \\
\hline 10 & Shrestha et al. (2016) & 2016 & Quality & Wheat & Camera & Kernels & ANN \\
\hline 11 & Singh and Chaudhury (2016) & 2016 & Quality & Rice & Camera & Kernels & BPNN \\
\hline 12 & Olgun et al. (2016) & 2016 & Quality & Wheat & Camera & Kernels & SVM \\
\hline 13 & Zareiforoush et al. (2015) & 2015 & Quality & Rice & Camera & Kernels & Fuzzy \\
\hline 14 & Kezhu et al. (2014) & 2014 & Quality & Soybean & Camera & Kernels & BPNN \\
\hline 15 & Sun et al. (2014) & 2014 & Quality & Rice & Camera & Kernels & SVM \\
\hline 16 & Ebrahimi et al. (2014) & 2014 & Quality & Wheat & Scanner & Kernels & ANN \\
\hline 17 & Serranti et al. (2013) & 2013 & Quality & Wheat & Hyperspectral & Kernels & iPLS-DA \\
\hline 18 & Naik et al. (2017) & 2017 & Phenotyping & Soybean & Camera & Plant & SVM \\
\hline 19 & Sadeghi-Tehran et al. (2017) & 2017 & Phenology & Wheat & Camera & Field & SVM \\
\hline 20 & Zhu et al. (2016) & 2016 & Phenology & Wheat & Camera & Field & SVM \\
\hline 21 & Lu et al. (2016) & 2016 & Phenology & Maize & Camera & Field & ANN \\
\hline 22 & Lu et al. (2015) & 2015 & Phenology & Maize & Camera & Field & SVM \\
\hline 23 & Guo et al. (2015) & 2015 & Phenology & Rice & Camera & Field & SVM \\
\hline 24 & Kurtulmuş and Kavdir (2014) & 2014 & Phenology & Maize & Camera & Field & SVM \\
\hline 25 & Romualdo et al. (2014) & 2014 & Phenology & Maize & Scanner & Leaves & Bayes \\
\hline
\end{tabular}


layers: convolutional layers, subsampling layers, and fully connected layers. Initially, LeNet-5 was used to recognize hand-written digits represented by two-dimensional $32 \times 32$-pixel images. The last layer contains the nodes that correspond to the probabilities of the original image belonging to one of the ten digit classes (Kirk and Hwu, 2016). Other network architectures are described in the literature: AlexNet (2012) (Krizhevsky et al., 2012), ZF Net (2013) (Zeiler and Fergus, 2013), VGG Net (2014) (Simonyan and Zisserman, 2015), GoogLeNet (2015) (Szegedy et al., 2015), Microsoft ResNet (2015) (He et al., 2016) are some of the examples. Some R-CNN (Region Based CNNs) networks are intended to detect objects by identifying regions that are most likely to contain the object to be identified. In this group are R-CNN (2013) (Girshick et al., 2014), Fast R-CNN (2015) (Girshick, 2015) and Faster R-CNN (2015) (Ren et al., 2015). From these architectures it is still possible to use them in combination to obtain more expressive results.

\section{Computer vision and agriculture}

The papers were grouped into three groups: diseases and pests, grain quality and phenology and phenotyping. Table 1 shows a summary of the main information about each paper.

\subsection{Grain diseases and insect infestations}

Grain production is susceptible to a number of adverse factors related to the occurrence of diseases or infestation by pests and insects. Such factors may trigger negative effects on crop development or reduce grain quality and, consequently, its market price.

The detection and accurate identification of diseases in grain crops have great importance for their effective management in order to guarantee productive and sustainable agriculture. Rapid identification in a pest infestation situation, for example, ensures that the response can be efficiently delivered and the necessary control measures can be taken.

The diagnosis of plant diseases is usually performed visually and may present flaws due to its laborious and subjective nature. In this sense, work was carried out with the aim of proposing methods of computer vision with artificial intelligence to automate the process of detection of diseases in plants. The automatic detection of diseases from images includes, among other factors, the determination of the most discriminative characteristics for the efficient recognition of the disease. Thus, you can use classification models to categorize an image into a healthy or sick class. However, when one considers the detection by images obtained in the field, one of the challenges is the presence of complex backgrounds and, consequently, the presence of interferences in the scene like occlusion, soil, plants of other species, specular reflection, among others (Barbedo, 2016).

In case of pest detection, traps can be used to improve the quality of the image segmentation and accurate the process to count the insects being in the scene. However, this method requires the previous management of that species. In addition, the coloration of some species are very close to those of the plant and, in this case, make it difficult to identify them. Finally, it is important to also emphasize the variability of the size and shape of these insects due to their species or stage of development.

\subsubsection{Bakanae detection in rice crops}

Chung et al. (2016) proposed a method to classify three-week-old seedlings in healthy and infected with Bakanae disease.Bakanae is a rice (Oryza sativa L.) disease caused primarily by the fungus Fusarium fujikuroi. When infected, plants exhibit color changes in the leaves (they become more yellowish), or morphological abnormalities such as stem elongation, stunting, or a greater angle between the leaves and the stem. The pathogen infects the grains in the fields and, during storage, multiplies itself by contaminating the seeds and spreading in the crop. The symptoms present great complexity and variability as they vary according to the resistance of the plant. In this way, they make detection through computer vision a challenge. The presented method uses plant images obtained using a scanner. The morphological and color attributes are quantified and the anatomical points of the plants are automatically identified. The only exception is the stem base that needs manual identification. In the preprocessing step, the background is removed using a thresholding operation. In the processing stage, morphological and coloring attributes are obtained to describe the development of the plant and indirectly provide its physiological status. SVM (Support Vector Machine) is used for the classification process. Two classifiers arranged in cascade are used to detect and determine the health condition of the plant. The first classifier is responsible for distinguishing between healthy and contaminated plants. The second classifier has the function of distinguishing the different levels of contamination.

4.1.2. Computer vision methods aptitude to detect leaf diseases in soybeans Shrivastava et al. (2016) explored the aptitude of computer vision methods to detect and categorize soybean plant foliar diseases. In the study, the following descriptors were analyzed: histogram; WDH (Wavelet Decomposed Color Histogram); BIC (Border/Interior Classification), which consists of a compact method to characterize each pixel of the image as border or interior; the CCV (Color Coherence Vector), which allows the comparison of images using their color density; the $\mathrm{CDH}$ (Color difference histogram), which allows the visual appearance of the images to be coded; the LBP (Local binary pattern) descriptor, which is used to encode image texture information; the SSLBP descriptor (Square Symmetric Local Binary Pattern), similar to the previous one, however, aiming to reduce the size of the LBP; the LAP (Localized angular phase), which calculates the magnitude and the phase of the Fourier transform of the neighbors of each pixel and serves to encode texture information; and the SEH (Strutucture Element Histogram), which encodes both color and texture information. For the classification, three models were analyzed: SVM, KNN (k-Nearest Neural Network) and PNN (Probabilistic Neural Network). Among the main results, it can be highlighted that BIC, histogram, and WDH presented the best performances in the detection of soybean leaf diseases analyzed in the study. The CDH and LAP descriptors were more robust for unfocused images. Finally, among the classification methods utilized, the best performance was obtained by KNN and SVM.

\subsubsection{Aphids detection in wheat}

Aphids are one of the pests that affect the wheat crop. These insects feed on the sap of the wheat phloem affecting its development and can transmit a series of viral pathologies. Currently, the most widely used method for identifying and counting is still the manual. In order to monitor populations of aphids and to identify the species present in the field, Liu et al. (2016) proposed the SMH method (Support Vector Machine, Maximally Stable Extremely Regions and Histograms of Oriented Gradient Method). The method combines the use of SVM, the MSER (Maximally Stable Extremal Regions) algorithm, and HOG (Histogram of Gradients). The images used for training the classification model were obtained using conventional cameras positioned at a lateral angle to the plants during the period between the elongation and emergency stages. In the pre-processing step, filters are used to enhance contrast and reduce noise. In this step, the use of the MSER algorithm aims to simplify the background of the images. In the extraction step of the image attributes, the HOG descriptor is obtained to be used as a parameter for classification using SVM for the presence or absence of aphids. The proposed method was tested with different densities of aphids, color or location in the plant. The SMH was compared with five other methods commonly used in the detection of aphids using three species that differ in their coloration. The results demonstrated superior performance for aphid detection. 


\subsubsection{Local descriptors to detect soybeans diseases}

Pires et al. (2016) proposed a method of automated disease detection in soybean. The method is based on local descriptors and the BOV (Bag of Values) method for encoding the input vectors for the classifier. The leaves were obtained in soybean plantations in Brazil. After collected in the field those leaves were scanned in order to generate images. The use of local descriptors proves to be robust to occlusion and do not require segmentation of the image to be used. The method evaluated five different descriptors: SURF (Speeded-Up Robust Features), HOG, DSIFT (Dense Scale-invariant Feature Transform), SIFT (Scale-invariant Feature Transform) and PHOW (Pyramid histograms of visual words). For the classification, two classes were defined, sick and healthy, in an SVM classifier.

\subsubsection{Detection and evaluation of leaf spot severity in wheat}

Han et al. (2015) proposed a computer vision method to automatically identify diseases that cause leaf spot in rice. The images were obtained in the field, so it was necessary to treat the background objects. Due to the noise present in the images, it is critical first to extract the target plant and then to identify which disease patterns are present in its leaves. For extraction of the attributes of the image, the first operation performed is the segmentation. To separate the target sheet from the background, the authors used the MCW (Marker-Controlled Watershed) algorithm. Once the target sheet is identified, it is necessary to obtain the characteristics of the disease that may be present in it. For this purpose, the SLIC algorithm (Simple Linear Iterative Clustering) Superpixels was used because it groups the pixels of the image into small regions with some meaning. The attributes selected for classification are texture (obtained using GLCM (Gray Level Co-occurrence Matrix)), gradient, Gabor, and attributes inspired by biology. One of the reasons for including gradient features was the nonuniformity of illumination of the artifacts. The classification model adopted for the diagnosis of diseases was the SVM. For the severity measurement, the Dice coefficient was used. The results were compared with a simple neural network. In all cases, the proposed method presented better performance.

\subsubsection{Sitophilus granarius detection in stored wheat grains}

Another pest that can cause extensive damage to stored grains in silos is the Sitophilus granarius, also known as "granary weevil". Detection of this type of infestation can be difficult and usually requires the presence of a specialist, increasing the time and cost of this type of assessment. Boniecki et al. (2014) proposes to identify the presence of this insect in grains. The method consists of using images obtained with a low-energy X-ray apparatus, in order to identify internally damaged grains. The grains are arranged on X-ray plates to produce the images. These are initially binarized using the thresholding operation. Conventional methods of image analysis are used to identify some attributes such as grain circumference, area, vertical and horizontal size. From these values, it is possible to calculate the attributes that will be used as input for the classification model. The eight attributes selected by the authors were: non-dimensional shape coefficient, Feret coefficient, two proportions of circularity, Malinowska coefficient, area, circumference, and cultivar. The neural model selected for classification is the MLP (Multi-Layer Perceptron). The configuration of the network that presented the best results was the one that uses three layers. This configuration has obtained the highest number of properly sized damaged grains. The sensitivity analysis of the neural network helped to identify the order of importance of grain identification characteristics. Cultivation, Feret coefficient, and area of the grain were the most important for the detection of the presence of infestation in wheat grain.

\subsection{Grain quality}

Grain quality is a complex phenomenon influenced by several genetic and environmental factors. Conventional optical techniques adopted by the industry for grading wheat grains usually present high misclassification error rates(Serranti et al., 2013). In this sense, the use of machine learning techniques for image classification has been highlighted in recent years as a way to build more accurate and intelligent classification systems. However, unlike other industrial products, the size, color, and texture of agricultural products are not defined by a single mathematical function making the task of classification challenging for computer vision systems.

Computer vision systems are an alternative to manually inspecting grain samples. Information on grain type and quality are required at various stages during the grain processing process. Contrary to expectations, however, visual inspection of these products is tedious and time-consuming. The operator usually loses concentration after hours of work due to fatigue, tired vision or inadequate lighting. Thus, an automatic classifier can prevent human errors in the quality assessment process, making it an alternative to manual inspection (Singh and Chaudhury, 2016).

\subsubsection{Classification of wheat grains using Artificial Neural Network}

Sabanci et al. (2017) presented a computer vision system that uses a simplified classification approach with a high index of accuracy. The aim of the system is to classify wheat grains of the species Triticum aestivum and Triticum durum according to their visual characteristics using an artificial neural network of the MLP type. The images are obtained by a camera at an angle perpendicular to the grain. Then those images are converted to grayscale, binarized using the Otsu method and segmented using the thresholding operation. The characteristics of size, color and texture are captured for each grain, with the purpose of serving as input to the classification method. In the study, seven visual characteristics of the grain were selected: length, length and width ratio, green, blue, green ratio, homogeneity, and entropy. The last two, concerning texture, are obtained using the GLCM method. The ANN (Artificial Neural Network) based on MLP with three layers was able to classify in bread wheat and durum wheat.

\subsubsection{Recognition of fungal colonies in rice using computer vision and machine learning techniques}

Sun et al. (2016) investigated the potential of using computer vision with conventional and deep learning techniques of machine learning applied with detection of mould colonies in unhulled paddy caused by microorganisms such as Aspergillus and Penicillium. The system for obtaining the images consists of a camera, two LED strips, a camera holder and a holder for the sample on a black base. In the pre-processing step, the black background of the images is removed and the size of images normalized. The center pixel coordinates are calculated to eliminate interference from the plate used for sample deposition. The identification of the infected regions is done through the color information of the image. For this, two images of $512 \times 512$ pixels are generated: one in grayscale and one in RGB. The vector of 64 color attributes is obtained from the 16-level histogram for the grayscale image and for each of the color channels of the RGB image. The areas where the fungus is present in the sample images do not have a uniform color, texture or shape. Due to this fact, the segmentation method using threshold is useless for the segmentation of the areas of interest. In order to deal with these limitations, the PSR (Pitch Segmentation Recognition) method was adopted for segmentation. The use of the SPA (Successive Projection Algorithm) algorithm was evaluated to reduce the number of attributes of the images from 64 to 14 . The attributes number is validated using the RMSECV (Root Mean Square Errors of Cross Validation) method. Classification methods such as SVM, BPNN (Back-Propagation Neural Network), CNN (Convolution Neural Network) and DBN (Deep Belief Network) models are compared. Although the DBN method presents the best results in the study, the SVM model can obtain good results for different classification tasks in agriculture since the values obtained are slightly lower than the DBN model. The SVM model depends on the support vectors that are extracted from the input data. Within the 
number of training samples, the number of support vectors of the SVM method also increases, raising a complexity of the model. Finally, the BPNN model shown inappropriate when the number of input variables was large.

\subsubsection{Shadow-based method to quantify the percentage of filled rice grains}

As stated by Liu et al. (2016) rice grain filling is a critical factor in determining grain yield. They presented a method for calculating the percentage of rice grains filling using shades. The method uses four light sources to generate grain shadow in four directions. The difference between the shadows of the filled grains and the unfilled grains is evaluated through image analysis and an SVM classifier. The system was designed to be used as an online assessment method. For this, a grain transport belt is used that allows the analysis at a speed of 40 to 50 grains/s. In the pre-processing step, the RGB images of the seeds with the shadows in the four directions are segmented using binarization. From the binarized image, you can extract information such as grain area and shadow area. Plus, two measurements are also determined: the ratio of the circumscribed rectangular area of the shadow and the ratio of the distance between the centroid and the circumscribed rectangle of the shadow. Both measurements can be used to distinguish filled grains from unfilled grains. The classification model adopted is the SVM. The results indicated that the method is reliable and can be used as a way of rapid evaluation of rice grains. As the filled grains are thicker than the unfilled ones, the shade of these will be wider. In this way, it is possible to use a sorter to separate the grains.

\subsubsection{A computer vision approach using two cameras to identify germinated wheat grains}

High moisture before or during harvesting can lead to premature germination of wheat grain. This condition causes a reduction in grain quality. Shrestha et al. (2016) propose a computer vision system that uses two cameras to separate and identify germinated wheat grains in controlled space. The system allows classification into three categories: healthy grains, damaged by germination and severely damaged by germination. The image acquisition system consists of two cameras: one positioned at the top and one positioned at the base. The segmentation technique adopted is MCW. The Watershed algorithm always produces closed object contours in addition to being computationally feasible. The approach of the use of markers aims to deal with the problem of the exaggerated segmentation of objects due to nature and the noise present in the images. It is also important to avoid grains not being overlapped to improve the accuracy of the grading method. The images produced by the two cameras are used to extract the morphological and non-morphological characteristics of the grains. The morphological characteristics used are perimeter, elliptic eccentricity, length of the main axis and relation of the length of the convex hull of the grain with its perimeter. The non-morphological characteristics used are RGB color, grayscale standard deviation, and texture. Grains damaged by germination usually have a yellow, green or dark brown coloration and may also have a whitish coloration. Such color changes are not present in healthy grains. To improve the accuracy of the classification method, it is important to avoid overlapping grain in the image. For classification, an artificial neural network was used that receives the 16 input parameters.

\subsubsection{Classification of rice grains using BPNN and wavelets}

Singh and Chaudhury (2016) propose the use of BPNN neural networks and decomposition using wavelets to classify rice grains. The approach uses two types of cameras to acquire the images: the first, present in cell phones that have a resolution of 0.3 megapixels and the second, a conventional point-and-shoot that has resolution of 12megapixel. The images are obtained under the condition of natural light in the morning to reduce shadow interference in the image. The extraction of attributes from the images is performed using three approaches. The first consists of extracting 18 color attributes (RGB and
HSI). The second and third approaches are to use the GLCM method and the wavelet-based decomposition for each color channel (R, G and B), respectively. The GLCM method is intended to handle texture attributes. These refer to a connected set of pixels that occur repeatedly in an image and are related to the intensity variation on a surface. For the description of texture attributes, the most accepted models are those based on the use of co-occurrences. In the discrete wavelet transform, an image signal can be analyzed using a set of filters followed by a decimation operation. The classification model uses a four-layer BPNN neural network. The study also compares the proposed method with other classifiers: SVM, k-nearest neighbor and naive Bayes. Among the other results obtained, we can highlight three. The first shows that it was necessary to use an adaptive thresholding function to ensure the convergence of the network. The second one points out that the approach that uses GLCM table demands greater processing than the one based on wavelets. And, finally, the use of BPNN presented better results when compared to the other methods.

\subsubsection{Wheat grains classification using DSIFT and SVM}

Olgun et al. (2016) evaluate the performance of using DSIFT technique in conjunction with an SVM classifier to classify wheat grains in 40 different species. SIFT is a computer vision algorithm to represent and identify objects with some differentiated local attribute. The DSIFT algorithm is derived from the SIFT algorithm. The main difference between them is that DSIFT uses a sampling procedure to reduce the cost of SIFT. Initially, the authors approach extracts the DSIFT attributes from the image. Following, the method of k-means is used on this set of attributes. Finally, the BOW model is constructed from the histogram of the clustering attributes obtained in the previous step. The objects of interest are classified, using the BOW model, through an SVM classifier. Prior to constructing the BOW model, the size of the set of attributes obtained by DSIFT should be reduced to optimize processing and improve accuracy. In this sense, the method of k-means clustering was applied.

\subsubsection{Classification of rice using a fuzzy inference system}

Zareiforoush et al. (2015) have used computer vision and fuzzy inference methods in the construction of a decision support system for quality classification of polished rice. The system uses two quality indices for the classification of images captured in a computer vision system: the degree of milling (DOM) and the percentage of broken kernels (PBK). The approach captures the images in RGB and segments the rice grains using thresholding. The PBK measure is obtained from the grain length in the images. The DOM measurement consists of evaluating the gray gradient. By the intensity of the grayscale, it is possible to evaluate the amount of light passing through a sample of rice. The greater the amount of bran remaining on the grain surface, the lower its translucency. To evaluate the amount of light, the co-occurrence matrix of a gray gradient is extracted from the images. This matrix shows the intensity distribution on a grayscale for each pixel of an image and what are the spatial relationships to each other. The DOM and PBK attributes are used as input to the Fuzzy Inference system. The join function maps the input values into five classes: very low, low, medium, high, and very high. The combination of the five classes of each attribute gave rise to 25 fuzzy rules of evaluation. In the defuzzification step, the method of calculating the area centroid is used to obtain the value of the sample quality. The system was superior when compared to the expert judgment.

\subsubsection{Identification of diseases in soybean seeds using BPNN}

Kezhu et al. (2014) proposed a machine vision method combined with BPNN to identify diseases in soybean seeds such as soybean frogeye, mildewed soybean, worm-eaten soybean and damaged grains. The acquisition of the images is performed from a specific device for the experiment. This device consists of a box of size $100 \mathrm{~cm} \times 30 \mathrm{~cm} \times 30 \mathrm{~cm}$ that does not allow the penetration of outside 
light. At its base is placed a black fabric that prevents light reflection. The system has a light-coupled ring-shaped light source capable of distributing light equally in all directions. The camera is positioned $60 \mathrm{~cm}$ above the grains, allowing the acquired images to be sharper, more accurate and easier to analyze. The system also has an illuminometer to measure the intensity of light. The METS (Minimum Error Threshold Selecting) method is used to separate the background of the image. Holes and noises are treated by means of morphological operations. In the end, the Watershed algorithm is used to handle cases where the beans are very close. The characteristic extraction step is to obtain morphological, color and texture parameters of the grains. The PCA (Principal Component Analysis) algorithm is used to reduce the vector dimensionality of the image attributes from 39 to 12 dimensions while maintaining $99 \%$ of the primary data from the experiment.

\subsubsection{Analysis of milled rice grains using SVM}

Chalkiness is an important factor to determine rice quality. Adverse factors such as harvesting immature grains and high moisture can affect it in a negative way. Usually, the evaluation is performed by manual inspection. However, in order to improve the efficiency of the quality process, the percentage of chalked grains should ideally be measured from grains randomly distributed in a sample. A challenge for automatic detection is the connected grains that are invariably present in the sample and separating them manually would significantly reduce process efficiency. Sun et al. (2014) proposed an automated system for analysis of the percentage of grains in rice. The algorithm deals with the separation of grains automatically. After segmentation, it is possible to obtain the number of grains present in the image and the specific information of the grain. It is known that the chalked areas are easily detected by the gray coloration. However, when the germ is not extracted completely the accuracy of the method may be affected. To deal with this situation, the system detects the location of the likely area of germ and considers this information in the classification process. Grain classification is done using the SVM method.

\subsubsection{Automatic wheat purity measurement system}

Ebrahimi et al. (2014) present a computer vision system that uses neural networks and an imperialist algorithm to automatically detect the purity of wheat. The experimental data used consist of 52 parameters of color, morphology, and texture extracted from images. The algorithm combines ICA (Imperialist Competitive Algorithm) and ANN to achieve two purposes: to find the set of best parameters and to create a robust classification system. The system is trained to classify wheat grains and impurities, In the first one, the elimination of shadows using the RGB and HSI color spaces and mathematical operations of the images is carried out by means of a table scanner. The selection of attributes that characterize the objects of interest is one of the most significant challenges in pattern recognition. purpose of the feature selection process is to identify the ideal number of attributes that generate the expected results in the algorithms This process may be non-intuitive because parameters that perform poorly when used separately may have better results when used together. ICA is used as a way to optimize the number of extracted features.

4.2.11. Development of a method using hyperspectral images for detection of wheat grains damaged by Fusarium, mottled and vitreous

Serranti et al. (2013) developed a method based on the classification of hyperspectral images for identification of three types of wheat (Triticum durum): vitrified, yellow berry and damaged by Fusarium. Vitreous grains are hard, translucent and amber. Non-vitreous grains are opaque and soft. The vitreous degree is, therefore, an important quality factor for wheat because it improves the quality of the flour and the color of the foods produced with it. In contrast, yellow berry or mottled grains adversely affect the quality of the flour produced because of its yellowish color and the soft grain appearance. However, the mixing of predetermined quantities of mottled and vitrified grains is tolerated by the industry. Grains affected by Fusarium have low market interest and, consequently, reduced final sales price. This is due to concerns about the food safety of wheat because the contamination caused by this type of fungus causes in the presence of mycotoxins in the grain. Image acquisition consists of obtaining hyperspectral images of selected wheat grains in the laboratory using a device equipped with an HSI (Hyperspectral Imaging) system operating in the near-infrared range $(1000-1700 \mathrm{~nm})$. The PCA technique is used for exploratory purposes to analyze the common characteristics of the samples and their groups: samples characterized by similar spectra can be grouped into the same product class. However, PCA is an unsupervised method and cannot be used to construct a predictive model, such as for classifying samples. In this sense, the PLS-DA (Partial Least Squares Discriminant Analysis) technique is used to identify the model capable of classifying the types of wheat used. The spectral data obtained from the wheat images present a high degree of dimensionality due to the redundancy between continuous variables (wavelengths). Therefore, it is necessary to select a reduced set of wavelengths to optimize the processing. The iPLS-DA (Interval Partial Least Squares Discriminant Analysis) method allows selection of a reduced subset of wave intervals. Initially, the authors obtain 121 wavelengths, which are reduced to 92 in order to minimize the effect of the noise caused by the background. With the use of the iPLS-DA technique, this number is reduced to 12 wavelengths. Comparing the results obtained for the different grades, the best grading model was the grading obtained for vitreous grains.

\subsection{Phenology and phenotyping}

Phenology is the branch of ecology that studies the events that occur during the life cycle of the plant in reaction to the conditions of the environment (Fina, 1973). Temperature, light, and humidity are some of the examples of environmental conditions that can trigger reactions such as flowering and fruiting in plants. The stage of development of the plant, for example, is one of the attributes that, in most cases, is evaluated manually. This task, like grain inspection and disease assessment, is laborious and susceptible to human failure (Zhu et al., 2016). The application of a computer vision system, in this case, would offer a productive and non-invasive alternative to the manual observation work required in the process of plant phenotyping.

\subsubsection{Phenotyping framework using machine learning to evaluate the severity of stress in soybean plants}

Naik et al. (2017) present a real-time phenotyping framework using computer vision and machine learning to classify the severity of iron deficiency chlorosis in soybean plants. The workflow presented for the phenotyping consists of the following steps: (a) capture the image; (b) storage and curation; (c) extraction of phenotypic characteristics; (d) machine learning (classification); (e) model for use in decision support systems. In the study, ten approaches to classification are compared in order to select the best approach for use in a smartphone application. The images were obtained using a DSLR camera and calibrated using the X-Rite ColorChecker. In the pre-processing step, the white adjustment and the color calibration are performed to guarantee the uniformity of the collected images. In the segmentation step, the images are converted from RGB to HSV with the objective of efficient removal of the black background using the technique of thresholding. The noise and the remaining isolated points are eliminated using the component connection method. This method consists of detecting clusters of pixels that are connected to each other to identify the largest component. According to the protocol for the acquisition of images, the plant should be positioned in the center, so the removal of the unconnected components is simplified. The extraction of characteristics consists in calculating the percentage of yellow, corresponding to the regions of chlorosis, and the percentage of brown, corresponding to the regions of necrosis of the plant. In the classification stage, the authors compare ten models: decision trees, random forests, naive Bayes, linear discriminant 
analysis, quadratic discriminant analysis, SVM, k-nearest neighbors and Gaussian mixture model. Accuracy and interpretability (end-user ability to interpret output data) were evaluated for the construction of a smartphone application that included both the processing and the classification of the images. The chosen method taking into account these aspects was the hierarchical model composed of two stages of classification by means of the SVM method.

\subsubsection{Automatic method to determine wheat emergence and flowering} stages

Sadeghi-Tehran et al. (2017) present an automated method to detect the flowering stage of wheat using a computer vision system. The method is not limited to specific wheat cultivars and therefore can be employed for a variety of cultivars without any adjustment necessary. The method is robust to handle lighting differences and natural lighting conditions in the field. It is robust, too, in the distinction of newly emerged ears, despite its similarity of color between leaves and ears. The technique is performed in four steps: (a) acquisition of image through a camera; (b) image pre-processing for contrast adjustment using the DS (Decorrelation Stretching) technique; (c) extraction of attributes; (d) classification of images. The DS method consists of widening the color differences and increasing the contrast of the image by removing the correlation between channels found in each pixel. It is possible, in this way, to distinguish the spikes from the leaves. The method lets you highlight details in images, such as spikes, even when they are difficult for identification with the naked eye. In the attribute extraction step, the SIFT and BOV methods are used to generate the visual vocabulary of the image. The classifier adopted for the method is the SVM.

\subsubsection{Automatic observation of wheat development in the field}

Zhu et al. (2016) propose an automatic observation system for wheat spikelet development based on computer vision. Images are obtained under natural conditions with frequent changes of lighting directly from the field. To ensure that these are representative of the plot, a statistical analysis is performed. For the system to adapt to a complex environment, the detection mechanism of the proposed spike is formed by two steps: rustic and fine. The rustic detection step is to use the DS technique to highlight the regions of interest and an SVM classifier to find the regions containing the wheat ears. In the thin detection stage, for each region detected by the SVM classifier, DSIFT techniques are used to extract the attributes of the image and again to classify it using SVM. The FV technique (Fisher Vector) is used to optimize the attribute vector that will be used as input to the classification algorithm. The result is quite acceptable when compared to manual observations. The classification performed using two-step SVM was compared to other methods such as ExGExR (Excess Green - Excess Red) color index, saliency, multiple color, and k-Means. The method excels in the majority of cases. The ExGExR and saliency methods are not suitable for the detection of wheat spikelets since they do not perform well into complex backgrounds.

\subsubsection{Corn tassel characterization}

Lu et al. (2015) present a computer vision system called mTassel for the purpose of characterizing the corn tassel using a computer vision system. This technique combines multiple views of attributes and color channels in order to reduce the influence of environmental variations. In addition to the detection of the total number of tassels, other characteristics such as tassel color, number of branches, length, width, perimeter and diameter are also obtained to allow monitoring over time. A large database (10 sequences with 16,031 samples) was used. The results of the work can be used for the detection of the stage of development, accurate estimation of production and dismantling by machines, as well as future research involving phenotyping. The images are captured hourly, between 9am and $4 \mathrm{pm}$, by a camera installed in the field. The ground truth is obtained by manually annotating the attributes of the corn tassel. The system consists of four modules: P, D, S and $\mathrm{T}$. The first, module $\mathrm{P}$, is responsible for detecting the potential regions that can hold the banners in the images. Module D is responsible for the accurate detection of the tassel in the set of regions previously determined. The module $S$ extracts the tassel shape attributes. Finally, the $\mathrm{T}$ module estimates the shape parameters and number of banners. Mathematically, any color space can be viewed as a kind of linear or non-linear transformation of RGB space. Considering the fact that there is a margin of difference between colors, the goal is to look for a method that can distinguish one color from another. Machine learning techniques are used to identify the linear projection that best distributes colors in a three-dimensional space. Thus, they can be used to convert RGB to a novel color space called SLC (Saliency Color Space). The colors that correspond to the tassel are improved, while the background colors are compressed and restricted. After the step of thresholding, the appearance of noise is common. Therefore, a filtering operation is normally used to reduce noise. Intuitively, the method can be seen with a "high pass" filter with a frequency threshold computed automatically. The unpredictable variation of the scene, for example, occlusion and specular reflection, may break the detection area of the tassel. However, a potential detection area must be continuous. Thus, the Euclidean distance transform is introduced to connect the separated areas and complete the proposed region. In module D, SVM classifiers are used for the detection of banners. From an image classified as being of a tassel, the module $S$ extracts and delimits the shape of the tassel in the image. In this module again an SVM classifier is used to name the banding and background regions. The t module is detected and delimited, the $\mathrm{T}$ module extracts the attributes of color, number of branches, width, perimeter, diameter and number of banners. The results obtained by the method showed the superiority of the segmentation module when compared to the methods AP-HI (Yu et al., 2013), LCM (Teixidó et al., 2012) and HSeg (Tang et al., 2011).

\subsubsection{Maize tassel segmentation based on the region-based color modeling method}

Lu et al. (2016) present a method for segmentation of the maize tassel capable of processing different colors and, thus, enable its use in several cultivars. The approach adopted by the method uses the technique of segmentation based on graphs and the algorithm SLIC. The first has the effect of softening the limits of the regions while the second, SLIC, preserves the borders of the region. From these identified regions, the method proposes which image color modeling will be used by neural networks. This model is called NNI (Neural Network Intensity). Instead of training the classifier using the entire sample set, the model trains a neural network for each image intensity, in order to achieve greater robustness to the lighting modifications in the scene. The results demonstrate that the method achieves a favorable performance and that the approach used has sufficient flexibility and expandability to be applied in other cultures. The method overcomes the state of the art regarding the segmentation of the corn tassel. The NNI model, when compared to a traditional neural network, improves the overall performance of the method. The mTassel-S (Lu et al., 2015) method presented inferior performance when used with the test images.

\subsubsection{Automatic characterization of rice flowering}

Guo et al. (2015) present an automated system for the characterization of rice flowering dynamics using a series of RGB images acquired in the field. According to the authors, flowering (anthill of the spikelet) is one of the most important phenotypic characteristics of rice and, consequently, considerable effort is expended by the researchers to observe its dynamics. Extrusion of the anthers lasts approximately one to two and a half hours daily during the breeding phase and the external characteristics of the environment are highly sensitive. The proposed method uses RGB, DSIFT, BOV and SVM images. The module installed in the field consists of a camera and microcontroller programmed to monitor the power, control the camera trigger and send the images to a 
server via Wi-Fi. The system initially monitors few samples due to its high cost. However, the method can be extended to be used on drones. Due to the size of the rice flower, image resolution affects the performance of the method. Low-resolution images have a negative impact on the accuracy of the method. The used SVM classifier obtained 83\% accuracy when using 30 images for training and 64\% accuracy when 300 images were used in contradiction to what was expected. Normally it is expected that when using a larger number of images for training a classifier this will perform better. One of the reasons that may explain the fact is that the complexity of the background generates high variation in the SIFT descriptors and, consequently, the more images for the training, the greater the impact on the accuracy. However, according to the authors, further studies still need to be done to determine the real cause of the problem.

\subsubsection{Corn tassel detection using computer vision and SVM}

Dismantling corn is a task that can be performed manually or with the help of machines. However, the success rate normally achieved by machines in this process ranges from $60 \%$ to $90 \%$. Yet, when employed, there is a need for people to complete the process, as the desired success rate is $99 \%$. The main problem in this scenario is that in corn plantations the height of the plant is not constant and, even in machines that use optical sensors, a more accurate discharge has not yet been reached. Kurtulmuş and Kavdir (2014) present a study to explore the possibility of determining the cutting position of the corn tassel in a plantation using computer vision. The method can be summarized in the following sequence of steps: (a) obtaining RGB images; (b) binarization of images using an SVM classifier; (c) application of morphological operations in the image; (d) construction of distance map; (e) identification of the potential position of the spikes. In binarization of the image, the SVM classifier used is trained to detect pixels belonging to the banners with the color information as input in the RGB, HSI and YCbCr spaces. The result of binarization consists of several connected parts and other unconnected parts belonging to both the tassel and regions of image noise. In this sense, morphological operations in the image are necessary to reduce the search regions and to extract the potential regions for tassel location. The result is non-uniform and uneven areas. The next step is to calculate the Euclidean distance of each pixel from the foreground to the nearest pixel of the background and thus obtain a distance map. In this phase of the algorithm, potential locations for the tassel include false positives. To eliminate them, the SIFT and GLCM techniques are used to extract the shape and texture attributes respectively. The consolidation of these results allows obtaining a potential tassel location and potential cut position.

\subsubsection{Diagnosis of nutritional status relative to nitrogen in corn leaves}

Romualdo et al. (2014) propose a computer vision method to detect the nutritional status of nitrogen in maize. Identification of nutritional status using leaf chemistry requires leaf sampling when the plant is in an advanced stage of development. In this way, it impedes the corrective action in the crop in course, if the detection of deficiency of a specific nutrient is detected. The main objective of the system is to identify nutritional deficiencies at different stages of plant development, especially in the early stages of growth, which may contribute to the early diagnosis and correction in the same growth cycle. For the acquisition of images, a table scanner is used. The first steps of the algorithm are to remove the background of the image, separate the scanned sheets and orient them horizontally. Several small regions of the leaves are extracted for texture analysis. These regions undergo a statistical analysis to determine if it is representative to use them for sheet representation. If they are not, they are discarded. The set of texture attributes extracted are VFD (Volume Fractal Dimension), GW (Gabor Wavelet) and VFDCA (VFD with Canonical Analysis). Classification is performed using the Bayes method. Analyzing the results obtained, the use of the GW method presented better performance.

\section{Discussion}

In general, the methods analyzed use the same organization: image acquisition, processing, and classification. However, each situation requires the selection of the adequate algorithms considering the individual characteristics of the problem that will be handled. There is no default workflow that can be applied in a generic way to the cultures without adjustments in its strategy of detection and processing of images.

The segmentation of images also plays an important role in achieving the desired result. Although it is simple to use for images obtained in controlled environments, this task is not trivial when images are obtained directly from the crop. In such a situation, the lighting variability, the complexity of the background, and the position of image capture are some of the factors that make this task challenging. In this scenario, the use of artificial intelligence already in the segmentation stage can bring positive results to the general accuracy of the method. Another great challenge to be addressed by the algorithms is the variability of the physiological characteristics of the plants because, when combined, they increase even more the diversity of factors that need to be analyzed in the image processing.

Machine learning algorithms are sensitive to the volume and quality of information used to train them. In some situations, when the algorithm "learns" about noise and excessive details of the input set, we have the overfitting situation that negatively impacts the generalization capacity of the built model. However, when the data is not sufficient, the model may not be able to generalize when used with validation data. The evaluated works showed different approaches to deal with this situation and properly select the attributes that guarantee the best performance of the method. In addition, in order to guarantee good performance, it is necessary to reduce the dimensionality of the input vectors for the classification algorithms, in order to optimize the processing of the learning algorithms. The SVM classifier, used in $56 \%$ of the analyzed works, can be pointed at the predominant technique, having good results of accuracy and performance. In spite of this, because it is only able to classify data into two groups, the approaches studied end up using a hierarchical structure to increase the greater number of classes. It should be noted that algorithms such as DBN and $\mathrm{CNN}$, although recent and with few case studies, present a promising alternative for processing large volumes of complex data.

The implementation of a considered set of works utilized algorithms for processing in CPU and modules available in the MATLAB platform. No evidence was found of the use of large-scale GPUs in the implementation of the analyzed works. In contrast, it is understood that recent advances in this area are promising in raising the processing and classification capacity of pattern recognition models. Deep Neural Networks has become a viable solution for image classification thanks to the development of the computational power of GPUs in recent years (Coates et al., 2009). Besides that, CNN has stood out as one of the most effective techniques in pattern recognition applications with a large number of images (Lee et al., 2015). There is a great advantage in using this kind of hardware since deep neural networks processing exploits the features in which the GPUs stand out: high intensive data parallel operations.

Interest in the use of computer vision and artificial intelligence was evident. Recent works like automatic diseases detection and identification (Lu et al., 2017; Ferentinos, 2018), microorganisms classification (Kosov et al., 2018), detection of stored-grain insects using deep learning (Shen et al., 2018) are some of the examples. Classification systems are tools that also aid the grain marketing processes. These systems aim to meet the needs of buyers and at the same time encourage producers to seek the desired quality as they provide equitable returns in relation to what is being marketed. Handling, transportation, processing, treatment, and storage operations are significantly influenced by the characteristics of the grain classification system adopted. Grains produced and harvested in the field are physically 
heterogeneous due to the diversity of the other elements present as plants, insects, inert matter, and soil (Hulasare et al., 2003). Therefore, due to its importance in the grain production chain, it is evident the need for agile and effective methods for its utilization. In this sense, computer vision presents as a valuable asset.

Two other aspects are explored in the literature: crop monitoring and cultivar identification. Cultivar identification is an important aspect in the development of a new cultivar. Cultivar development requires a great deal of effort in the genetic improvement process and requires a large number of crosses. During this process, it is essential for the breeder to precise control which progenies are being tested and which crosses are being carried out. Therefore, it is essential the protection of the cultivar generated to guarantee to the breeder the expected commercial return relative to this intense work accomplished. One of the necessary conditions for the protection of a cultivar is to evaluate whether the material meets the technical criteria of distinguishability, homogeneity, and stability (Act 1991,). Another aspect related to cultivar identification is the importance of correct cultivar selection to the producer. The selection of the cultivar to be used by the producer is an important decision that considers, mainly, economic factors, maturity, and resistance to diseases. The purity of the cultivar should also be considered. Some of the criteria used to differentiate cultivars may be influenced by the environment and in some cases, these characteristics are phenotypically very similar. In this context, the use of advanced techniques for the differentiation of cultivars involving laboratory tests through the use of electrophoresis and chromatography can be avoided if efficient non-destructive methods of cultivar identification are available. Last, crop monitoring has gained evidence since it opens the possibility of integration with other automation technologies involving robotics and autonomous vehicles. This point was not explored in the present work but some results like corn tassel identification show valuable when applied in conjunction with automated machines.

Manual methods for grain assessment are challenging even for people who are able to perform these tasks. One of the negative aspects is the time required to carry out such assessments, which impedes rapid decision-making and large-scale evaluation (Zareiforoush et al., 2015). However, due to the natural variability of the products, the task of identification and classification is extremely challenging and computationally intensive because it involves a large number of data due to the number of parameters to be used in the classification (Visen et al., 2002).

In the context of cultivar identification, the selection of the cultivar that will be used by the producer is an important decision that takes into account economic factors, maturity, resistance to diseases, among other characteristics. The purity of the cultivar to be chosen should also be considered. Some of the criteria used to differentiate cultivars may be influenced by the environment and in some cases, these characteristics are phenotypically very similar. In this context, the use of advanced techniques for the differentiation of oat cultivars involving laboratory tests through the use of electrophoresis and chromatography is justifiable.

Consider, for example, the three oat cultivars UPFA 20 - Teixeirinha, UPFA 21 - Moreninha, UPFA 22 - Temprana. Teixeirinha and Temprana are cultivars indicated for food industry consumption with a highquality standard (Universidade de Passo Fundo and de Aveia, 2017). However, the cultivar Temprana has a super early cycle, while Teixeirinha has a regular cycle. Moreninha is a black oat cultivar, suitable for grazing and ensiling. Therefore, the latter has a completely different application of the first two. Therefore, it is understood that there is a demand for fast methods to identify cultivars at different points along the oat production chain, from the stage of creation and registration of new cultivars, through planting and finishing with grain processing by the industry. In addition, the quality of the grains is also an important aspect evaluated by the industry. In this situation, a manual approach requires time and specific laboratories available.

\section{Conclusion}

Computer vision systems are already widely employed in different segments of agricultural production and industrial food production. They can be used in grading systems for orange, papaya, almond, potato, lemon, wheat, corn, rice, and soybean. Its use is justifiable due to the benefits obtained. The use of such systems provides a simple and objective analysis of the samples, producing accurate descriptive data. Through these systems it is possible to automate laborious tasks, in a non-destructive way, producing adequate data for future analysis.

It was identified that there are gaps to be filled with the development of intelligent devices that use computer vision and artificial intelligence for automation of tasks in the field, as well as their integration with agricultural machines and drones. Expanding the use of GPUs and advanced artificial intelligence techniques are also promising alternatives for future work. Wheat, oat and barley cultivars, for instance, could benefit from a computer system that aims to reduce complexity and costs in the gluten-containing grains classification from images.

Finally, our intention that this survey would present diverse applications and techniques of machine learning, image and video processing in order to motivate more researchers to apply them for solving agricultural problems currently open. The study of the presented works and the problems that they set out to solve together with the new advances in computer vision and artificial intelligence can lead to new solutions for agriculture bringing gains of production, quality, and food security.

\section{References}

ACM Digital Library, 2018. < https://dl.acm.org/>.

Act 1991, 1991. International convention for the protection of new varieties of plants. <http://www.upov.int/upovlex/en/conventions/1991/act1991.html >.

Barbedo, J.G.A., 2013. Digital image processing techniques for detecting, quantifying and classifying plant diseases. SpringerPlus 2 (1), 660. https://doi.org/10.1186/21931801-2-660. <http://springerplus.springeropen.com/articles/10.1186/2193-1801$2-660>$

Barbedo, J.G.A., 2016. A review on the main challenges in automatic plant disease identification based on visible range images. Biosyst. Eng. 144, 52-60. https://doi. org/10.1016/j.biosystemseng.2016.01.017. < <ttp://www.sciencedirect.com/ science/article/pii/S1537511015302476>

Boniecki, P., Piekarska-Boniecka, H., Świerczyński, K., Koszela, K., Zaborowicz, M., Przybył, J., 2014. Detection of the granary weevil based on x-ray images of damaged wheat kernels. J. Stored Prod. Res. 56, 38-42. https://doi.org/10.1016/j.jspr.2013. 11.001. < http://www.sciencedirect.com/science/article/pii/ S0022474X13000829>

Breuel, T.M., Ul-Hasan, A., Al-Azawi, M.A., Shafait, F., 2013. High-performance ocr for printed english and fraktur using lstm networks. In: 2013 12th International Conference on Document Analysis and Recognition, pp. 683-687. doi:https://doi. org/10.1109/ICDAR. 2013.140.

Chung, C.-L., Huang, K.-J., Chen, S.-Y., Lai, M.-H., Chen, Y.-C., Kuo, Y.-F., 2016. Detecting Bakanae disease in rice seedlings by machine vision. Comput. Electron. Agric. 121, 404-411. https://doi.org/10.1016/j.compag.2016.01.008. < http://www. sciencedirect.com/science/article/pii/S0168169916000132>

Coates, A., Baumstarck, P., Le, Q., Ng, A.Y., Scalable learning for object detection with GPU hardware. In: 2009 IEEE/RSJ International Conference on Intelligent Robots and Systems, IEEE, pp. 4287-4293. doi:https://doi.org/10.1109/IROS.2009.5354084. <http://ieeexplore.ieee.org/document/5354084/>

Davies, E. (Ed.), 2005. Machine Vision, third ed., Signal Processing and its Applications, Morgan Kaufmann, Burlington. doi:https://doi.org/10.1016/B978-0-12-206093-9. 50039-3. < http://www.sciencedirect.com/science/article/pii/ B9780122060939500393>

Ebrahimi, E., Mollazade, K., Babaei, S., 2014. Toward an automatic wheat purity measuring device: a machine vision-based neural networks-assisted imperialist competitive algorithm approach. Measurement 55, 196-205. https://doi.org/10.1016/j. measurement.2014.05.003. < <ttp://www.sciencedirect.com/science/article/pii/ S0263224114002097>

Ferentinos, K.P., 2018. Deep learning models for plant disease detection and diagnosis. Comput. Electron. Agric. 145, 311-318. https://doi.org/10.1016/J.COMPAG.2018. 01.009. <https://www.sciencedirect.com/science/article/pii/ S0168169917311742\#f0005>

Fina, A.L.d., 1973. Climatologia y fenologia agricolas. first ed., EUDEBA.

Food, 2012. A.O. of the United Nations, Faostat database. < http://www.fao.org/ faostat $>$.

Girshick, R.B., 2015. Fast R-CNN. In: 2015 IEEE International Conference on Computer Vision, ICCV 2015, Santiago, Chile, December 7-13, 2015, pp. 1440-1448. doi:https://doi.org/10.1109/ICCV.2015.169.

Girshick, R.B., Donahue, J., Darrell, T., Malik, J., 2014. Rich feature hierarchies for 
accurate object detection and semantic segmentation. CoRR abs/1311.2524. $<$ http://arxiv.org/abs/1311.2524>

Guo, W., Fukatsu, T., Ninomiya, S., 2015. Automated characterization of flowering dynamics in rice using field-acquired time-series RGB images. Plant Meth. 11 (1). https://doi.org/10.1186/s13007-015-0047-9. < http://plantmethods. biomedcentral.com.ez103.periodicos.capes.gov.br/articles/10.1186/s13007-0150047-9>.

Han, L., Haleem, M.S., Taylor, M., 2015. A novel computer vision-based approach to automatic detection and severity assessment of crop diseases. In: 2015 Science and Information Conference (SAI), pp. 638-644. doi:https://doi.org/10.1109/SAI.2015. 7237209.

He, K., Zhang, X., Ren, S., Sun, J., 2016. Deep residual learning for image recognition. In: 2016 IEEE Conference on Computer Vision and Pattern Recognition, CVPR 2016, Las Vegas, NV, USA, June 27-30, 2016, pp. 770-778. doi:https://doi.org/10.1109/ CVPR.2016.90.

Hornberg, A., 2017. Handbook of Machine and Computer Vision, Wiley-VCH Verlag GmbH \& Co. KGaA. doi:https://doi.org/10.1002/9783527413409.fmatter.

Hulasare, R., Dronzek, B., Jayas, D., 2003. Grain-Grading Systems. CRC Presshttps://doi. org/10.1201/9780203911310.ch3.

IEEE Xplore Digital Library, 2018. < https://ieeexplore.ieee.org/Xplore/home.jsp > .

Kamilaris, A., Prenafeta-Boldú, F.X., 2018. Deep learning in agriculture: a survey. Comput. Electron. Agric. 147, 70-90. https://doi.org/10.1016/J.COMPAG.2018.02. 016. <https://www.sciencedirect.com/science/article/pii/ S0168169917308803>

Kezhu, T., Yuhua, C., Weixian, S., Xiaoda, C., 2014. Identification of diseases for soybean seeds by computer vision applying BP neural network. Int. J. Agric. Biol. Eng. 7 (3), 43-50. https://doi.org/10.3965/ijabe.v7i3.1028. < <ttps://ijabe.org/index.php/ ijabe/article/view/1028>

Kirk, D.B., Hwu, W.-m.W., 2016. Programming Massively Parallel Processors, Third Edition: A Hands-on Approach. third ed. Morgan Kaufmann Publishers Inc., San Francisco, CA, USA.

Kitchenham, B., Charters, S., 2007. Guidelines for performing systematic literature reviews in software engineering.

Kosov, S., Shirahama, K., Li, C., Grzegorzek, M., 2018. Environmental microorganism classification using conditional random fields and deep convolutional neural networks. Pattern Recogn. 77, 248-261. https://doi.org/10.1016/J.PATCOG.2017.12. 021. < https://www.sciencedirect.com/science/article/pii/ S0031320317305174>

Krizhevsky, A., Sutskever, I., Hinton, G.E., 2012. Imagenet classification with deep convolutional neural networks. In: Pereira, F., Burges, C.J.C., Bottou, L., Weinberger, K. Q. (Eds.), Advances in Neural Information Processing Systems 25, Curran Associates, Inc., pp. 1097-1105. http://papers.nips.cc/paper/4824-imagenet-classification-withdeep-convolutional-neural-networks.pdf.

Kurtulmus, F., Kavdir, İ., 2014. Detecting corn tassels using computer vision and support vector machines. Expert Syst. Appl. 41 (16), 7390-7397. https://doi.org/10.1016/j. eswa.2014.06.013. < http://www.sciencedirect.com/science/article/pii/ S0957417414003546>

Lecun, Y., Bottou, L., Bengio, Y., Haffner, P., 1998. Gradient-based learning applied to document recognition. Proc. IEEE 86 (11), 2278-2324. https://doi.org/10.1109/5 726791.

Lee, S.H., Chan, C.S., Wilkin, P., Remagnino, P., 2015. Deep-plant: Plant identification with convolutional neural networks. In: 2015 IEEE International Conference on Image Processing (ICIP). IEEE, pp. 452-456. https://doi.org/10.1109/ICIP.2015. 7350839. <http://ieeexplore.ieee.org/document/7350839/>.

Liu, T., Chen, W., Wu, W., Sun, C., Guo, W., Zhu, X., 2016. Detection of aphids in wheat fields using a computer vision technique. Biosyst. Eng. 141, 82-93. https://doi.org/ 10.1016/j.biosystemseng.2015.11.005. < http://www.sciencedirect.com/science/ article/pii/S1537511015300866>

Liu, T., Wu, W., Chen, W., Sun, C., Chen, C., Wang, R., Zhu, X., Guo, W., 2016. A shadowbased method to calculate the percentage of filled rice grains. Biosyst. Eng. 150, 79-88. https://doi.org/10.1016/j.biosystemseng.2016.07.011. < http://www. sciencedirect.com/science/article/pii/S1537511016300174>

Lu, H., Cao, Z., Xiao, Y., Fang, Z., Zhu, Y., Xian, K., 2015. Fine-grained maize tassel trait characterization with multi-view representations. Comput. Electron. Agric. 118, 143-158. https://doi.org/10.1016/j.compag.2015.08.027. < http://www. sciencedirect.com/science/article/pii/S0168169915002537>

Lu, H., Cao, Z., Xiao, Y., Li, Y., Zhu, Y., 2016. Region-based colour modelling for joint crop and maize tassel segmentation. Biosyst. Eng. 147, 139-150. https://doi.org/10. 1016/j.biosystemseng.2016.04.007. <http://www.sciencedirect.com/science/ article/pii/S1537511015304438>

Lu, J., Hu, J., Zhao, G., Mei, F., Zhang, C., 2017. An in-field automatic wheat disease diagnosis system. Comput. Electron. Agric. 142, 369-379. https://doi.org/10.1016/ J.COMPAG.2017.09.012. < https://www.sciencedirect.com/science/article/pii/ S0168169917305999\#f0005>

Mahajan, S., Das, A., Sardana, H.K., 2015. Image acquisition techniques for assessment of legume quality. Trends Food Sci. Technol. 42 (2), 116-133. https://doi.org/10.1016/ j.tifs.2015.01.001. < http://www.sciencedirect.com/science/article/pii/ S0924224415000023>

Marchi, E., Ferroni, G., Eyben, F., Gabrielli, L., Squartini, S., Schuller, B., 2014. Multiresolution linear prediction based features for audio onset detection with bidirectional lstm neural networks. In: 2014 IEEE International Conference on Acoustics, Speech and Signal Processing (ICASSP), pp. 2164-2168. doi:https://doi.org/10. 1109/ICASSP. 2014.6853982.

Marsland, S., 2014. Machine Learning: An Algorithmic Perspective, second ed. Chapman \& Hall/CRC.

Naik, H.S., Zhang, J., Lofquist, A., Assefa, T., Sarkar, S., Ackerman, D., Singh, A., Singh,
A.K., Ganapathysubramanian, B., 2017. A real-time phenotyping framework using machine learning for plant stress severity rating in soybean. Plant Meth. 13 (1), 23 https://doi.org/10.1186/s13007-017-0173-7. < <https://link.springer.com/article/ 10.1186/s13007-017-0173-7>

Olgun, M., Onarcan, A.O., Ö-zkan, K., Işik, Şahin, Sezer, O., Ö-zgişi, K., Ayter, N.G., Başçiftçi, Z.B., Ardiç, M., Koyuncu, O., 2016. Wheat grain classification by using dense sift features with svm classifier. Comput. Electron. Agric. 122, 185-190. https://doi.org/10.1016/j.compag.2016.01.033. < <ttp://www.sciencedirect.com/ science/article/pii/S0168169916300060>

Pires, R.D.L., Gonçalves, D.N., Oruê, J.P.M., Kanashiro, W.E.S., Rodrigues Jr., J.F., Machado, B.B., Gonçalves, W.N., 2016. Local descriptors for soybean disease recognition. Comput. Electron. Agric. 125, 48-55. https://doi.org/10.1016/j.compag. 2016.04.032. <http://www.sciencedirect.com/science/article/pii/ S016816991630179X>.

Prince, S.J.D., 2012. Computer Vision: Models, Learning, and Inference, first ed. Cambridge University Press, New York, NY, USA.

Ren, S., He, K., Girshick, R.B., Sun, J., 2015. Faster R-CNN: towards real-time object detection with region proposal networks. In: Advances in Neural Information Processing Systems 28: Annual Conference on Neural Information Processing Systems 2015, December 7-12, 2015, Montreal, Quebec, Canada, pp. 91-99. < http://papers. nips.cc/paper/5638-faster-r-cnn-towards-real-time-object-detection-with-regionproposal-networks $>$

Romualdo, L.M., Luz, P.H.C., Devechio, F.F.S., Marin, M.A., Zúñiga, A.M.G., Bruno, O.M., Herling, V.R., 2014. Use of artificial vision techniques for diagnostic of nitrogen nutritional status in maize plants. Comput. Electron. Agric. 104, 63-70. https://doi. org/10.1016/j.compag.2014.03.009. < <ttp://www.sciencedirect.com/science/ article/pii/S0168169914000696>

Russell, S., Norvig, P., 2009. Artificial Intelligence: A Modern Approach, third ed. Pearson. < http://www.amazon.com/exec/obidos/redirect?tag = citeulike07-20\& path $=$ ASIN $/ 0136042597>$.

Sabanci, K., Kayabasi, A., Toktas, A., 2017. Computer vision-based method for classification of wheat grains using artificial neural network. J. Sci. Food Agric. 97 (8), 2588-2593. https://doi.org/10.1002/jsfa.8080.

Sadeghi-Tehran, P., Sabermanesh, K., Virlet, N., Hawkesford, M.J., 2017. Automated method to determine two critical growth stages of wheat: heading and flowering. Front. Plant Sci. 8https://doi.org/10.3389/fpls.2017.00252. < http://journal frontiersin.org/article/10.3389/fpls.2017.00252/abstract > .

Sak, H., Senior, A.W., Beaufays, F., 2014. Long short-term memory based recurrent neural network architectures for large vocabulary speech recognition, CoRR abs/1402.1128. $<$ http://arxiv.org/abs/1402.1128>.

Schaeffel, F., Hornberg, A., 2017. Handbook of Machine and Computer Vision. Wiley VCH Verlag GmbH \& Co. KGaA. doi:https://doi.org/10.1002/9783527413409.ch1.

ScienceDirect, 2018. < https://www.sciencedirect.com/>.

Scopus, 2018. <https://www.scopus.com/freelookup/form/author.uri >

Serranti, S., Cesare, D., Bonifazi, G., 2013. The development of a hyperspectral imaging method for the detection of Fusarium-damaged, yellow berry and vitreous Italian durum wheat kernels. Biosyst. Eng. 115 (1), 20-30. https://doi.org/10.1016/j. biosystemseng.2013.01.011. <http://www.sciencedirect.com/science/article/pii/ S1537511013000196>

Shah, J.P., Prajapati, H.B., Dabhi, V.K., 2016. A survey on detection and classification of rice plant diseases. In: 2016 IEEE International Conference on Current Trends in Advanced Computing (ICCTAC), pp. 1-8. doi:https://doi.org/10.1109/ICCTAC.2016. 7567333.

Shen, Y., Zhou, H., Li, J., Jian, F., Jayas, D.S., 2018. Detection of stored-grain insects using deep learning. Comput. Electron. Agric. 145, 319-325. https://doi.org/10 1016/J.COMPAG.2017.11.039. < https://www.sciencedirect.com/science/article/ pii/S016816991730769X\#f0005>

Shrestha, B.L., Kang, Y.-M., Yu, D., Baik, O.-D., 2016. A two-camera machine vision approach to separating and identifying laboratory sprouted wheat kernels. Biosyst. Eng. 147, 265-273. https://doi.org/10.1016/j.biosystemseng.2016.04.008. < http:// www.sciencedirect.com/science/article/pii/S1537511015303433>.

Shrivastava, S., Singh, S.K., Hooda, D.S., 2016. Soybean plant foliar disease detection using image retrieval approaches. Multimedia Tools Appl. 1-28. https://doi.org/10 1007/s11042-016-4191-7. < <ttps://link.springer.com/article/10.1007/s11042016-4191-7>

Simonyan, K., Zisserman, A., 2015. Very deep convolutional networks for large-scale image recognition. CoRR abs/1409.1556. < http://arxiv.org/abs/1409.1556>.

Singh, K.R., Chaudhury, S., 2016. Efficient technique for rice grain classification using back-propagation neural network and wavelet decomposition. IET Comput. Vision 10 (8), 780-787. https://doi.org/10.1049/iet-cvi.2015.0486.

Springer Link, 2018. < https://link.springer.com/>

Sun, C., Liu, T., Ji, C., Jiang, M., Tian, T., Guo, D., Wang, L., Chen, Y., Liang, X., 2014. Evaluation and analysis the chalkiness of connected rice kernels based on image processing technology and support vector machine. J. Cereal Sci. 60 (2), 426-432. https://doi.org/10.1016/j.jcs.2014.04.009. < <ttp://www.sciencedirect.com/ science/article/pii/S0733521014000861>

Sun, K., Wang, Z., Tu, K., Wang, S., Pan, L., 2016. Recognition of mould colony on unhulled paddy based on computer vision using conventional machine-learning and deep learning techniques. Sci. Rep. 6, 37994. https://doi.org/10.1038/srep37994. < http://www-nature-com.ez103.periodicos.capes.gov.br/srep/2016/161129/ srep37994/full/srep37994.html>

Sutskever, I., Vinyals, O., Le, Q.V., 2014. Sequence to sequence learning with neural networks. In: Proceedings of the 27th International Conference on Neural Information Processing Systems - Volume 2, NIPS'14, MIT Press, Cambridge, MA, USA, pp. 3104-3112. < http://dl.acm.org/citation.cfm?id =2969033.2969173>.

Szegedy, C., Liu, W., Jia, Y., Sermanet, P., Reed, S.E., Anguelov, D., Erhan, D., Vanhoucke, 
V., Rabinovich, A., 2015. Going deeper with convolutions. In: IEEE Conference on Computer Vision and Pattern Recognition, CVPR 2015, Boston, MA, USA, June 7-12, 2015, pp. 1-9. doi:https://doi.org/10.1109/CVPR.2015.7298594.

Tang, W., Zhang, Y., Zhang, D., Yang, W., Li, M., 2011. Corn tassel detection based on image processing. In: International Workshop on Image Processing and Optical Engineering, Vol. 8335. doi:https://doi.org/10.1117/12.917672.

Teixidó, M., Font, D., Pallejà, T., Tresanchez, M., Nogués, M., Palacín, J., 2012. Definition of linear color models in the rgb vector color space to detect red peaches in orchard images taken under natural illumination. Sensors 12 (6), 7701-7718. https://doi. org/10.3390/s120607701. < http://www.mdpi.com/1424-8220/12/6/7701> .

Universidade de Passo Fundo, 2017. Cultivares de Aveia. <http://aveia.upf.br/> .

Visen, N., Paliwal, J., Jayas, D., White, N., 2002. Ae-automation and emerging technologies: specialist neural networks for cereal grain classification. Biosyst. Eng. 82 (2), 151-159. https://doi.org/10.1006/bioe.2002.0064. < http://www. sciencedirect.com/science/article/pii/S1537511002900644> .

Vithu, P., Moses, J.A., 2016. Machine vision system for food grain quality evaluation: A review. Trends Food Sci. Technol. 56, 13-20. https://doi.org/10.1016/j.tifs.2016.07. 011. < http://www.sciencedirect.com/science/article/pii/S092422441630084X> . Web of Science, 2018. <https://www.webofknowledge.com>.

Yu, Z., Cao, Z., Wu, X., Bai, X., Qin, Y., Zhuo, W., Xiao, Y., Zhang, X., Xue, H., 2013.
Automatic image-based detection technology for two critical growth stages of maize: emergence and three-leaf stage. Agric. For. Meteorol. 174, 65-84. https://doi.org/10. 1016/j.agrformet.2013.02.011. <http://www.sciencedirect.com/science/article/ $\mathrm{pii} / \mathrm{S} 0168192313000397>$.

Zareiforoush, H., Minaei, S., Alizadeh, M.R., Banakar, A., 2015. Potential applications of computer vision in quality inspection of rice: a review. Food Eng. Rev. 7 (3), 321-345. https://doi.org/10.1007/s12393-014-9101-z. < <ttps://link.springer. com/article/10.1007/s12393-014-9101-z> .

Zareiforoush, H., Minaei, S., Alizadeh, M.R., Banakar, A., 2015. A hybrid intelligent approach based on computer vision and fuzzy logic for quality measurement of milled rice. Measurement 66, 26-34. https://doi.org/10.1016/j.measurement.2015.01.022. <http://www.sciencedirect.com/science/article/pii/S0263224115000482>.

Zaremba, W., Sutskever, I., Vinyals, O., 2014. Recurrent neural network regularization. $<$ https://arxiv.org/abs/1409.2329>.

Zeiler, M.D., Fergus, R., 2013. Visualizing and understanding convolutional networks. CoRR abs/1311.2901. < http://arxiv.org/abs/1311.2901>.

Zhu, Y., Cao, Z., Lu, H., Li, Y., Xiao, Y., 2016. In-field automatic observation of wheat heading stage using computer vision. Biosyst. Eng. 143, 28-41. https://doi.org/10. 1016/j.biosystemseng.2015.12.015. < <ttp://www.sciencedirect.com/science/ article/pii/S1537511015300349> . 